\title{
Pinch Force Measurement in Minimally Invasive Surgery with Tactile Sensors
}

\author{
${ }^{1}$ Mithat Can Özin, ${ }^{* 2}$ Bilsay Sümer and $* 1$ İlker Murat Koç \\ ${ }^{1}$ Dept. of Mech. Eng., Istanbul Technical University, Gümüşsuyu, 34437, Beyoğlu, İstanbul, Turkey \\ *2Dept. of Mech. Eng., Hacettepe University, Beytepe, 06800, Ankara, Turkey
}

\begin{abstract}
This study focuses on the real-time measurement of the distributed pinch force in minimally invasive surgery with a piezoresistive tactile sensor. The measurement of the pinch force in distributed form is important because the conventional surgical tools have angular opening/closing mechanism, which causes non-uniform force distribution on the contacting sample. Additionally, the location and the magnitude of the equivalent pinch force is calculated. The grasping of the samples with homogenous and constant pinch force and the characterization of the samples with low elastic modulus can be carried out with tactile sensor included surgery tools. Apprentice surgeons can benefit the advantages of the thin film sensors in their training.
\end{abstract}

Key words: Tactile Sensor; Minimally Invasive Surgery; Laparoscopic Grasper

\section{Introduction}

In minimally invasive surgery, working space of the surgeon is limited and usage of the surgical tool reduces the tactile sensing of the surgeons. Apprentice surgeons are required to be trained until they learn how to use surgical tool without applying excessive forces [1-3]. Excessive force applied to tissue can cause tissue damage or rupture which is obligatory to be avoided [4-7]. Usage of tactile sensors to measure the pinch force can be used in training platforms or in surgical instruments. Thus, the surgeons are informed and aware. Most of the thin film tactile sensors are designed for flat-tipped laparoscopic graspers [8-11]. In this study, a piezoresistive tactile sensor specially manufactured for a flat-tipped bowel grasper is used to measure the pinch force applied to tissue in distributed form. Experiments are completed in ex vivo condition with organic samples made out of poultry.

\section{Materials and Method}

Details of tactile sensor and experimental setups are presented in this section.

\subsection{Tactile sensor specifications}

Measuring force in distributed form is a hard task to achieved and using a thin film sensor is one of the best decisions. Vertical and lateral resolution of the tactile sensors are important in measurement precision of the force and contact area, respectively [13]. Amount of tactile pixels

\footnotetext{
*Corresponding author: Address: Dept. of Mech. Eng., Hacettepe University, Beytepe, 06800, Ankara, TURKEY,
} 
(taxels) constraints the lateral resolution. A tactile sensor with 32 taxels in $8 \times 4$ grid is used in this study as illustrated in Fig. 1. Each taxel is $59.9 \mathrm{~mm}^{2}$. A signal conditioning unit is used with the tactile sensor. Moreover, a computer program is developed for real time measurement of the pinch force.

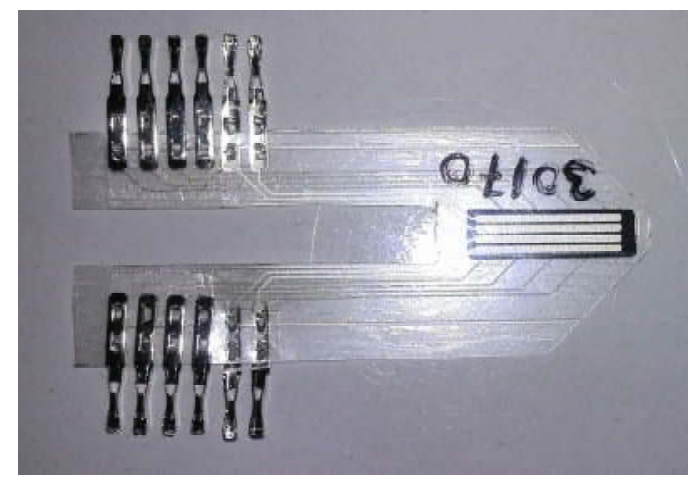

Figure 1. The tactile sensor with a $4 \times 8$ taxels grid.

\subsection{Experimental Setup}

This experimental setup shown in Fig. 2 is built to measure the pinch force of the tactile sensor integrated flat-tipped bowel grasper. Tactile sensor is placed into the tool tip. A linear motorized stage is connected to rod of the tool. Back and forth motion of the rod opens and closes the tip angularly while the tool is stationary. Due to angular motion of the tip, non-uniform force is applied to test samples. Non-uniform distributed pinch force is measured by tactile sensor then the location and magnitude of the equivalent pinch force is calculated in real time. Velocity of the rod is 0.5 $\mathrm{mm} / \mathrm{s}$ during closure and opening. Measurement range in this experimental procedure is below $0.7 \mathrm{~N}$. Experimental procedure consists of 4 steps. It starts by closure of the tip and achievement of the first contact between tip and sample. Subsequently, the maximum pinch force suddenly is achieved as soon as the maximum closure of the tip obtained. Force relaxation of the viscoelastic sample is noticed while the tip remains in maximum closure. Later, opening of the tip starts and the last contact between the tip and sample takes place.

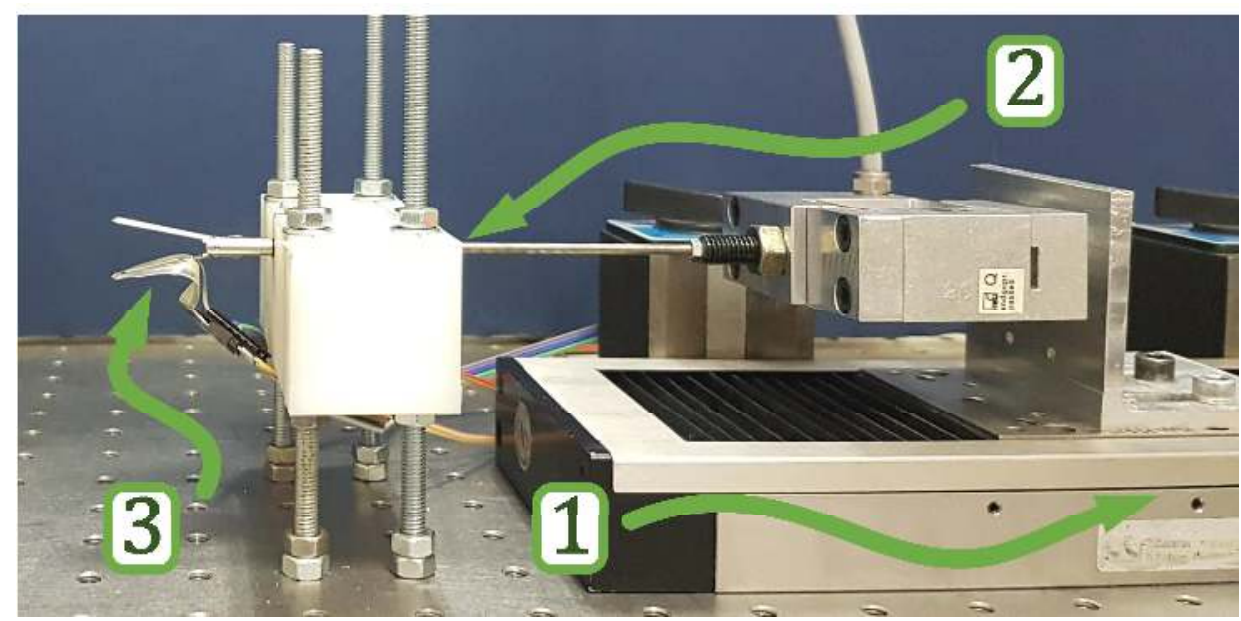

Figure 2. Experimental setup: (1) Motorized linear stage, (2) Flat-tipped tool, (3) Tactile sensor. 


\section{Results and Discussion}

Results of the pinch force measurement are presented in distributed and equivalent force form in Fig. 3 and Fig. 4, respectively. In Fig. 3, force distribution is given for two instants which are achievement of first contact (Fig. 3 (a)), achievement of maximum pinch force (Fig. 3 (b)). At the first contact, maximum taxel force magnitude is $0.03 \mathrm{~N}$. At maximum pinch force, maximum taxel force is $0.045 \mathrm{~N}$. Local forces are intense inwards of the tip and during the closure of the tip, local force distribution expands outwards of the tip due to the angular motion of the tip. Local pinch forces decrease gradually from inwards to outwards. Due to low elastic modulus of the sample, the sample deforms and full contact between the tip and sample can be achieved. From local pinch force data, the equivalent pinch force is calculated and in Fig 4., magnitude of the equivalent force is plotted versus time (a) and angle (b). Equivalent pinch force rises for $1.2 \mathrm{sec}$ during the closure of the tip and reaches to maximum value of $0.50 \mathrm{~N}$. After that, due to relaxation of the viscoelastic tissue pinch force declines while tip angle is constantly at minimum as seen in Fig.4 (b). Pinch force declines to zero with tip opening. Tactile sensors can measure relaxation characteristic of the sample in real time. With feedback control the sample can be grasped with constant pinch force. The location of equivalent pinch force is important to value to be calculated during the measurement of the pinch force. In Fig.5, the location of the equivalent pinch force from the same data of the Fig.3 and Fig.4 is presented. Closure of the tip is given between Fig.5 (a) and (d), opening of the tip is given between Fig.5 (e) and (f). During the closure, the location of the equivalent pinch force alternates from inwards of the tip to the middle of the tip as the pinch force expands more homogenously on tip surface. During the opening, it returns to inwards of the tip, as the pinch force concentrates again inwards of the tip.

(a)

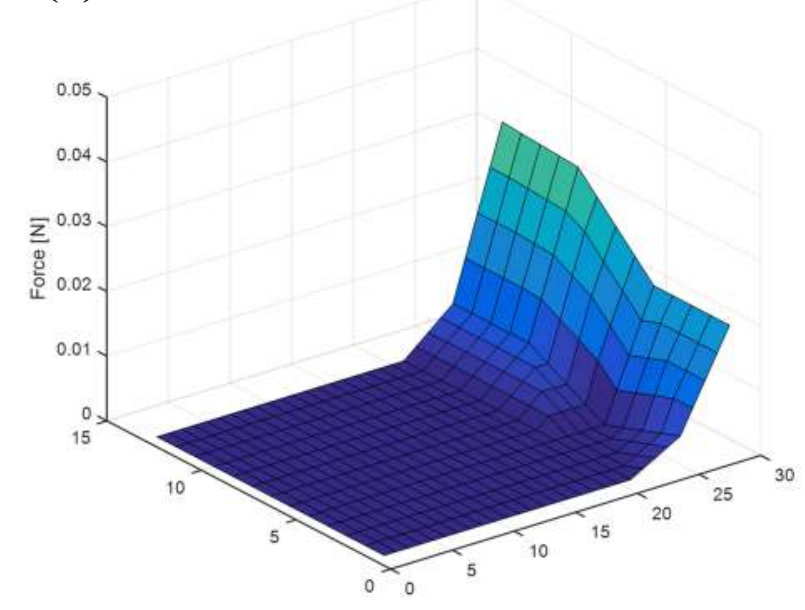

\section{(b)}

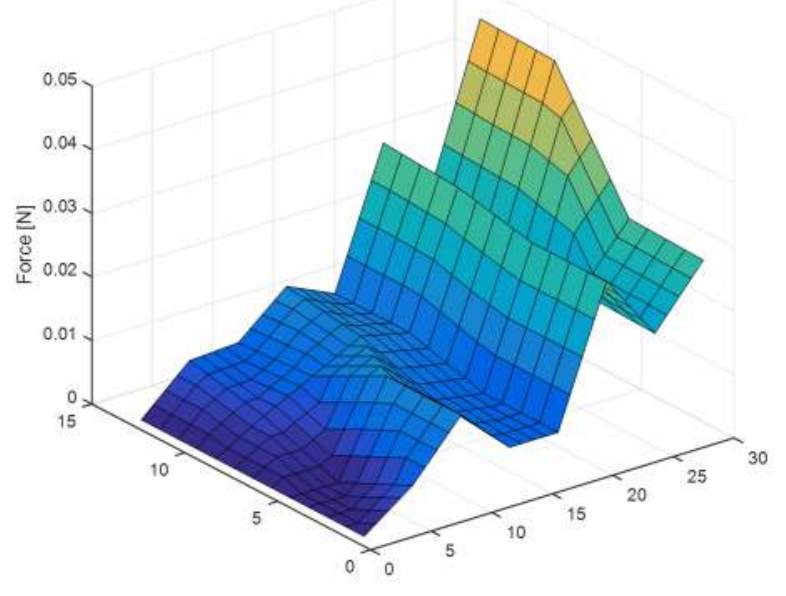

Figure 3. Force distribution at taxel level between tip and sample (a) First contact between tip and sample (b) Contact at the instant of maximum closure of the tip. $\mathrm{X}$ and $\mathrm{Y}$ axis indicate the location of the taxels placed on the tactile sensor and $\mathrm{Z}$ axis presents the force. 
(a)

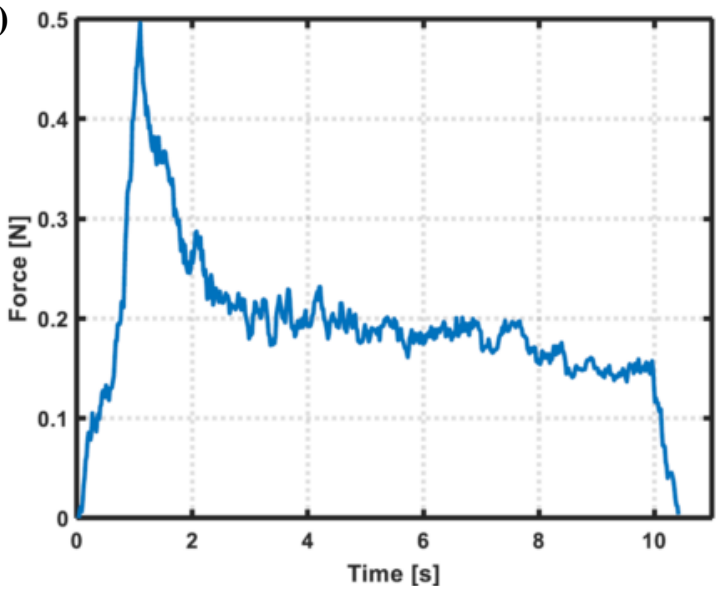

(b)

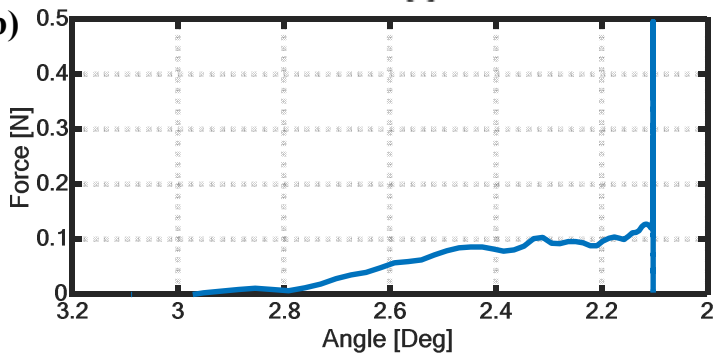

Figure 4. (a) Equivalent pinch force between tip and sample versus time. (b) Equivalent pinch force between tip and sample versus half angle of between tip, throughout closure tip angle decreases.

a

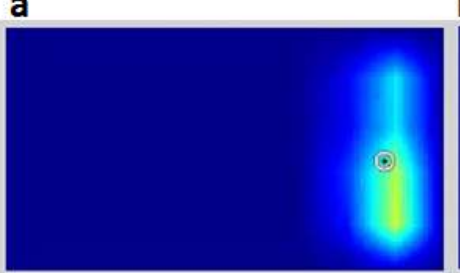

c

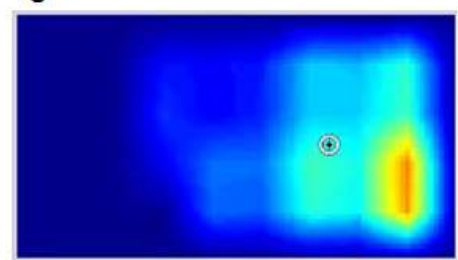

e

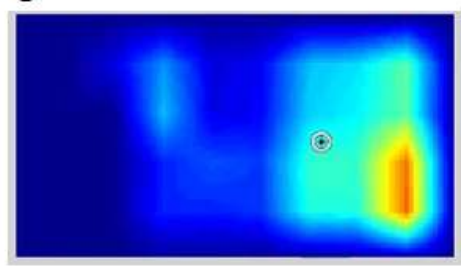

b

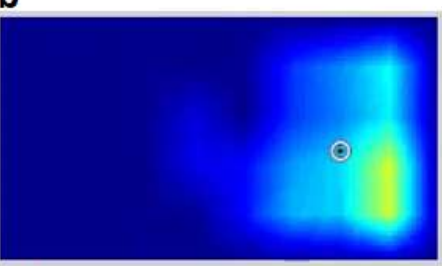

d

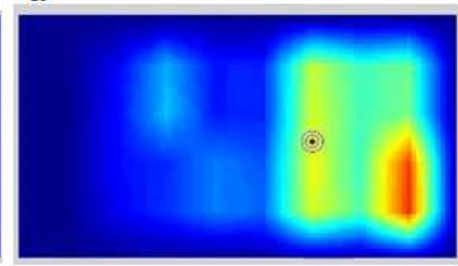

f

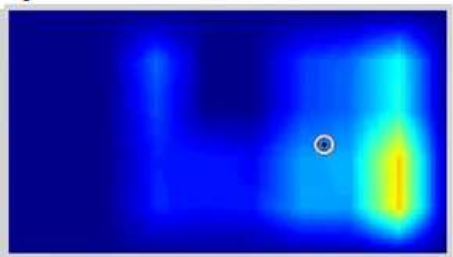

Figure 5. Location of the equivalent pinch force (white circle), (a) the first contact throughout the closure, (b) and (c) the continual closure of the tip (d) the instant of maximum closure, (e) the opening of the tip, (f) the last contact. 


\section{Conclusions}

This paper focuses on the measurement of the pinch force in minimally invasive surgery with tactile sensor. The measurement of the pinch force in distributed form is important because the conventional surgical tools have angular opening/closing mechanism, which causes non-uniform force distribution on the contacting sample. Local pinch forces are higher at inwards of tip and gradually decreases at outwards of the tip. Relaxation of the samples can be observed during the measurement of the equivalent pinch force. The location of the equivalent pinch force is closer to the inwards of the tip at the first contact. It shifts to the middle of the tip surface as the pinch force homogenously distribute on contact surface. The grasping of the samples with homogenous and constant pinch force and the characterization of the samples with low elastic modulus can be carried out with tactile sensor included surgery tools. Apprentice surgeons can benefit the advantages of the thin film sensors in their training. Prevention of the tissue damage can be avoided with force feedback control.

\section{References}

[1] Herder JL, Sjoerdsma WA. Laparoscopic Grasper with Force Perception. Science. 1997:5706;279-286. DOI=http://dx.doi.org/10.3109/13645709709153076.

[2] Vilos GA., Ternamian A, Dempster J, Philippe Y, Laberge PY, et al. Laparoscopic Entry: A Review of Techniques, Technologies, and Complications. Journal of Obstetrics and Gynaecology Canada. 2007;29(5):433-447. DOI=http://dx.doi.org/10.1016/S1701-2163(16)35496-2.

[3] Soper NJ, Brunt, LM, and Kerbl K. Laparoscopic general surgery. New England Journal of Medicine. 1994;330(6);409-419.

[4] Puangmali P, Althoefer K, Seneviratne LD, Murphy D, Dasgupta P. State-of-the-Art in Force and Tactile Sensing for Minimally Invasive Surgery. IEEE Sensors Journal 2008;8(4);371-381. DOI=http://dx.doi.org/10.1109/JSEN.2008.917481.

[5] Li W, Jia ZG, Wang J, Shi L, Zhou ZR. Friction Behavior at Minimally Invasive Grasper/Liver Tissue Interface. Tribology International. 2015:81;190-198. DOI=http://dx.doi.org/10.1016/j.triboint.2014.08.013.

[6] De S, Rosen J, Dagan A, Hannaford B, Swanson P, Sinanan M. Assessment of Tissue Damage due to Mechanical Stresses. The International Journal of Robotics Research. 2007:26;1159-1171. DOI=http://dx.doi.org/10.1177/0278364907082847.

[7] Cartmill JA, Shakeshaft AJ, Walsh WR, Martin CJ. High Pressures Are Generated At the Tip of Laparoscopic Graspers. ANZ Journal of Surgery. 1999:69(2);127-130. DOI=http://dx.doi.org/10.1046/j.1440-1622.1999.01496.x. 
[8] Sokhanvar S, Packirisamy M, Dargahi J. MEMS Endoscopic Tactile Sensor: Toward In-Situ and In-Vivo Tissue Softness Characterization. IEEE Sensors Journal. 2009:9(12);1679-1687. DOI=http://dx.doi.org/10.1109/JSEN.2009.2025586

[9] Hassanbeiglou A, Kalantari M, Mozaffari E, Dargahi J, Kövecses J. A New Tactile Array Sensor for Viscoelastic Tissues with Time-Dependent Behavior. Sensor Review. 2015:35(4);374-381. DOI=http:/dx.doi.org/10.1108/SR-06-2014-656.

[10] Peng P, Rajamani R, Erdman AG. Flexible Tactile Sensor for Tissue Elasticity Measurements. Journal of Microelectromechanical Systems. 2009:18(6);1226-1233. DOI=http://dx.doi.org/10.1109/JMEMS.2009.2034391.

[11] Koç İM, Akça E, Design of a Piezoelectric Based Tactile Sensor with Bio-inspired Micro/nanopillars. Tribology International.

DOI=http://dx.doi.org/10.1016/j.triboint.2012.06.003.

[12] Koç İM, Eray T, Sümer B, Çerç N. An Active Force Controlled Laparoscopic Grasper by Using a Smart Material Actuation. Tribology International. 2016:100;317-327. DOI=http://dx.doi.org/10.1016/j.triboint.2016.03.001.

[13] Sümer B, Aksak B, Şahin K, Chengsatiansup K, Sitti M, Piezoelectric Polymer Fiber Arrays for Tactile Sensing Applications. Sensor Letters. 2011:9(2);457-463. DOI=https://doi.org/10.1166/sl.2011.1498. 